\title{
Deterministic Rational Transducers and Random Sequences
}

\author{
Sylvain Porrot ${ }^{1}$, Max Dauchet ${ }^{2}$, Bruno Durand ${ }^{3}$, Nikolai K. Vereshchagin ${ }^{3,4}$ \\ ${ }^{1}$ LAIL, URA CNRS 1440 \\ Bâtiment P2, Université des Sciences et Technologies de Lille \\ 59655 Villeneuve d'Ascq CEDEX, France
}

Tel \& Fax : (33) 0320434743 - email : porrot@lifl.fr

${ }^{2}$ LIFL, URA CNRS 369

Bâtiment M3, Université des Sciences et Technologies de Lille

59655 Villeneuve d'Ascq CEDEX, France

Tel : (33) 0320434588 - email : dauchet@lifl.fr

${ }^{3}$ LIP, ENS-Lyon CNRS

46 Allée d'Italie

69634 Lyon CEDEX 07, France

e-mail : Bruno.Durand@ens-lyon.fr

${ }^{4}$ Dept. of Mathematical Logic and Theory of Algorithms

Moscow State University

Vorobjevy Gory, Moscow, Russia

e-mail : ver@mech.math.msu.su

\begin{abstract}
This paper presents some results about transformations of infinite random sequences by letter to letter rational transducers. We show that it is possible by observing initial segments of a given random sequence to decide whether two given letter to letter rational transducers have the same output on that sequence. We use the characterization of random sequences by Kolmogorov Complexity. We also prove that the image of a random sequence is either random, or non-random and non-recursive, or periodic, depending on some transducer's structural properties that we give.
\end{abstract}

\section{Introduction}

This paper starts the study of the deterministic rational transducers behaviour on infinite random sequences. Firstly, we show that it is possible by observing initial 
segments of a given random sequence to decide whether two given letter to letter rational transducers have the same output on that sequence (we call this problem equality problem). The analogous problem is undecidable on the class of all input sequences. Secondly, we prove that the image of a random sequence is either random or non-random and non-recursive or periodic, depending on the transducer's structural properties. Finally, we derive an arithmetical theorem from the previous result : the image of a 'random real' by a letter to letter rational transducer is either rational or transcendental.

This work is a part of a larger study on transformations processes of discrete curves. These processes are 'real time' transformations on random sequences. For us, 'real time' means that the computation time as well as the memory of the processes are bounded. Consequently, these processes are rational transducers [PD97].

The concept of random sequence has been well defined for the first time by MartinLöf in 1966 [ML66]. A sequence is random if and only if it does not belong to any constructive null set. Theory of Kolmogorov Complexity provides a characterization of such sequences in terms of compressibility. Levin and Schnorr have shown that the notions of random sequences and incompressible sequences are equivalent [Lev73] [Sch73]. This theory has enabled to obtain results on regular languages [Sei86] [CL88] [LV93], but our aim is different. The originality of this paper lies in the study of the effect of algebraic objects (rational transducers) on random sequences. This is another approach to Markov chains, which are probabilistic finite automata : here we consider deterministic automata and it is the input sequence that chooses the transitions. This choice is 'typically random' when the sequence is random.

We briefly present in section 1.1 the definition of random sequences due to Martin-Löf, and an equivalent definition in terms of Kolmogorov Complexity. There are several variants of this theory (see the foreword of Chaitin in [Cal94]), but here we consider only the Prefix Complexity. This variant is more convenient for the study of infinite sequences.

We consider classical rational transducers [MS97], introduced by M. Nivat [Niv68], reading infinite binary sequences in input and, unlike Büchi automata e.g. [Tho90], without any particular acceptance conditions. We do not require that for each state and each input letter there exists the corresponding transition. We say that a transducer accepts a sequence if it can read all its letters. In section 1.2, we introduce a classification of transducers' states. This classification is drawn from the theory of Markov chains [MT96]. The main aim of this classification is Lemma 5. It gives a necessary and sufficient condition of the acceptance of a random sequence.

In part 2, we show that the equality problem of two transducers is decidable on the class of random sequence, when it is undecidable on the class of all sequences. In order to 
accepts an infinite sequence if and only if both initial transducers accept this sequence and have the same output.

In part 3, Theorem 2 establishes a classification of the images of random sequences. This classification depends on the transducer's structural properties. On the one hand, the image of a random sequence is random if and only if there are not two paths with the same output linking two recurrent states. On the other hand, the image of a random sequence has finite complexity if and only if all paths starting from a recurrent state have the same output. Moreover, in this case, the image sequence is periodic.

Section 3.2 presents an application of the previous result within the frame of arithmetic. We call random real a real number of which the binary expansion is a random sequence. Theorem 3 claims that the image of any random real by a letter to letter rational transducer is either rational or transcendental.

\section{Preliminaries}

\subsection{Random sequences and Kolmogorov Complexity}

In this part we briefly present the definition of random sequences from Martin-Löf and the theory of Kolmogorov Complexity. In the following we call word (respectively sequence) any finite (respectively any infinite) string over the binary alphabet $\{0,1\}$.

\subsubsection{Random sequences}

The first satisfactory definition of a random sequence has been given by Martin-Löf in [ML66]. A sequence is random if and only if it does not belong to any constructive null set. More precisely, a sequence $s$ is non random if and only if there is a recursive function $u(i, j)$, mapping pairs of integers to words, satisfying the following properties :

1. $\forall i \mu\left(\Omega_{i}\right) \leq 2^{-i}$;

2. $\forall i s \in \Omega_{i}$.

where $\Omega_{i}=\bigcup_{j} \Gamma_{u(i, j)}, \Gamma_{u(i, j)}$ being the set of all the sequences having the prefix $u(i, j)$, and $\mu$ denotes uniform measure on the set of all sequences.

\subsubsection{Kolmogorov Complexity}

Theory of Kolmogorov Complexity [LV97], also called Algorithmic Information Theory, gives rigorous mathematical foundations to the notion of information quantity contained in an object $x$. This quantity $K(x)$ is the length of a smallest program computing $x$ without any input. The programming language must satisfy the following property : for any computable partial function $\phi$ mapping words to words there exists 
a constant $C$ such that for any $p$ in the domain of $\phi$ there exists a program computing $\phi(p)$ of length at most length $(p)+C$. We will use the variant of Kolmogorov Complexity called Prefix Complexity, in which the set of programs must be a prefix set: none of the programs is the prefix of another program. The complexity varies no more than by an additive constant when we change the programming language. Indeed,

$$
\exists C \forall x\left|K_{1}(x)-K_{2}(x)\right|<C
$$

where $K_{1}(x)$ and $K_{2}(x)$ are Kolmogorov complexities defined for two different programming languages.

\subsubsection{Randomness and incompressibility}

Theory of Kolmogorov Complexity provides a characterization of randomness using incompressibility properties. Actually Levin and Schnorr [Lev73] [Sch73] have shown that random sequences are exactly those incompressible for some variants of Kolmogorov Complexity. Their proof can be easily translated in the frame of Prefix Complexity that we use here. More specifically, all prefixes of a random sequence are compressible for less than a constant. Formally, if $a_{1: n}$ is the prefix of length $n$ of a sequence $a$, then this sequence is random if and only if :

$$
\exists c \forall n K\left(a_{1: n}\right) \geq n-c
$$

A random sequence is non-recursive, but non-random ones may be either recursive or non-recursive.

- Assume for example that the sequence $010011010110 \ldots$ is random. Then the sequence $001100001111 \ldots$, where each letter of the previous sequence appears twice, is non-random and non-recursive.

- The sequence $010101010101 \ldots$ is non-random and recursive.

In several proofs we show that a sequence $a$ is non-random building a program that computes $a$ using another sequence $a^{\prime}$ as an input. This program uses at most the $\lambda n$ first letters of $a^{\prime}$ to compute the $n$ first letters of $a$, with $\lambda<1$. The following lemma expresses this idea.

Lemma 1 Let $a$ and $a^{\prime}$ be two sequences. If there exists a program $P$ such that :

$$
\exists\left(u_{n}\right)_{n \in \mathbb{N}} \exists\left(v_{n}\right)_{n \in \mathbb{N}} \exists \lambda<1 \text { such that } \forall n P\left(a_{1: u_{n}}^{\prime}\right)=a_{1: v_{n}} \text { and } u_{n} \leq \lambda v_{n}
$$

then $a$ is non-random.

Proof We will give an upper bound of the complexity of the prefix words $a_{1: v_{n}}$ of $a$. The program $<P, a_{1: u_{n}}^{\prime}>$ computes $a_{1: v_{n}}$, but the set of these programs is not a prefix 
set. In order to obtain such a set we have to use the prefix coding of $a_{1: u_{n}}^{\prime}$, denoted by $\overline{a_{1: u_{n}}^{\prime}}$. For all $n$ we have $K\left(a_{1: v_{n}}\right) \leq\left|\overline{a_{1: u_{n}}^{\prime}}\right|+|P|+O(1)$. A classical upper bound for the prefix coding of a word $w$ is $|w|+2 \log (|w|)+O(1)$. Hence we have :

$$
\begin{aligned}
\forall n K\left(a_{1: v_{n}}\right) & \leq\left|a_{1: u_{n}}^{\prime}\right|+2 \log \left(\left|a_{1: u_{n}}^{\prime}\right|\right)+O(1) \\
& \leq \lambda\left|a_{1: v_{n}}\right|+2 \log \left(\left|a_{1: v_{n}}\right|\right)+O(1)
\end{aligned}
$$

Let $c>0$ be fixed. There exists $n_{c}$ such that :

$$
\forall n \geq n_{c} \lambda\left|a_{1: v_{n}}\right|+2 \log \left(\left|a_{1: v_{n}}\right|\right)+O(1) \leq\left|a_{1: v_{n}}\right|-c
$$

Eventually we have :

$$
\forall c \exists n_{c} \forall n \geq n_{c} K\left(a_{1: v_{n}}\right) \leq\left|a_{1: v_{n}}\right|-c
$$

Therefore $a$ is non-random.

\subsection{Transducers}

We consider classical transducers without final states since inputs are only infinite sequences. In this first approach we consider only letter to letter transducers : the output is a letter at each letter read in input. When the transducer reaches a state where there is no transition labelled by the input letter, it halts and we say that it rejects the input sequence. Otherwise it accepts the input sequence.

We give now some definitions and preliminary results considering only inputs on transitions.

Definition 1 Let $T$ be a transducer and a be a sequence accepted by $T$. A state of $T$ is recurrent for $a$ if it is reached an infinity of times with a as an input. $T_{a}$ denotes the set of recurrent states for $a$.

Definition 2 Let $q$ be a state of $T$. Let occ ${ }_{n}^{a}(q)$ denote the number of occurences of the state $q$ during the reading of $a_{1: n}$. The frequency $f^{a}(q)$ of $q$ is defined by :

$$
f^{a}(q)=\limsup _{n \rightarrow \infty} \frac{o c c_{n}^{a}(q)}{n}
$$

$A$ state $q$ is frequent if $f^{a}(q)>0$.

Definition 3 Let $q$ be a state of $T$ and $t$ be a transition from $q$. Let occ ${ }_{n}^{a}(t)$ denote the number of occurences of the transition $t$ during the $n$ first occurences of the state $q$ with $a$ as an input. The frequence $f^{a}(t)$ of $t$ is defined by:

$$
f^{a}(t)=\limsup _{n \rightarrow \infty} \frac{o c c_{n}^{a}(t)}{n}
$$


Lemma 2 Let a be a sequence accepted by $T . T$ contains at least one frequent state.

Proof Suppose that for each state $q_{i}$ of $T_{a}$ we have $f\left(q_{i}\right)=0$. Let $\epsilon=\frac{1}{2 Q}$ where $Q$ is the number of states of $T$. Therefore we have :

$$
\begin{aligned}
\exists n \forall q_{i} \in T \quad \frac{o c c_{n}^{a}\left(q_{i}\right)}{n} & \leq \epsilon \\
\sum_{i=1}^{Q} \frac{o c c_{n}^{a}\left(q_{i}\right)}{n} & \leq \epsilon Q \\
1 & \leq \frac{1}{2}
\end{aligned}
$$

Definition $4 A$ complete state is a state out of which get two transitions labelled by 0 and 1. We say that a set of states is complete if each state of this set is complete.

Claim 1 Consider the preorder on the set of the states of a transducer defined by :

$$
q<q^{\prime} \Leftrightarrow \text { we can reach } q^{\prime} \text { from } q
$$

and the equivalence classes of states. We say that maximal classes are absorbing. There is an algorithm that splits a transducer in sub-automata $T_{1}, \ldots, T_{N}$ and $T_{P}$ (See Figure 1. $T_{1}, \ldots, T_{N}$ are the complete absorbing classes and $T_{P}$ is the union of the remaining classes.

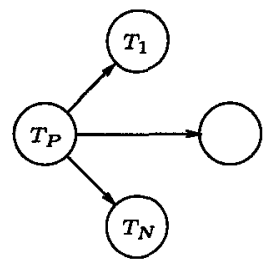

Figure 1: Complete absorbing classes

Lemma 3 Let a be a random sequence accepted by $T$. Any frequent state of $T$ is complete.

Proof Let $q$ be a frequent state. Suppose that $q$ is not complete. Thus, there is only one transition from this state, say the transition labelled by input letter 0 and leading to the state $q^{\prime}$. We show this implies that $a$ is non-random. Consider the sequence $a^{\prime}$ obtained as follows : in the sequence $a$ we delete letters read at each occurence of $q$. The following program computes $a$ using $T$ and $a^{\prime}$. 


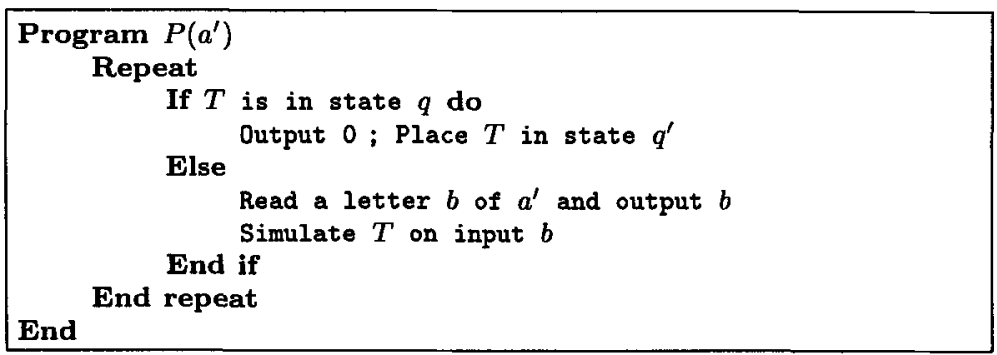

Since $q$ is frequent we have $\lim \sup _{n \rightarrow \infty} \frac{o c c_{n}^{\alpha}(q)}{n}=\alpha$ with $\alpha>0$. Thus there is a series $\left(v_{n}\right)_{n \in \mathbb{N}}$ and $n_{0}$ such that for all $n>n_{0}$ we have $\frac{o c c_{v_{n}}^{a}(q)}{v_{n}} \geq \frac{\alpha}{2}$. Let $u_{n}=v_{n}-o c c_{v_{n}}^{a}(q)$. For all $n>n_{0}$ we have $u_{n} \leq \lambda v_{n}$ with $\lambda=1-\frac{\alpha}{2}<1$. The program $P$ is such that for all $n$ we have $P\left(a_{1: u_{n}}^{\prime}\right)=a_{1: v_{n}}$. Thus hypothesis of Lemma 1 are satisfied, what enables us to conclude that $a$ is non-random.

Lemma 4 Let a be a random sequence accepted by $T$. Then all states of $T_{a}$ are frequent and $T_{a}$ is a complete absorbing class.

Proof According to Lemma 2, $T$ contains at least one frequent state $q$. This state belongs to $T_{a}$ and, according to lemma 3 , is complete. Let $t_{0}$ (respectively $t_{1}$ ) be the transition accepting 0 (respectively 1 ) and leading to $q_{0}$ (respectively $q_{1}$ ) from $q\left(q_{0}\right.$ and $q_{1}$ can be the same).

Step 1: We show that both transitions $t_{0}$ and $t_{1}$ are frequent. Suppose for example that $t_{0}$ is not frequent, i.e. $f^{a}\left(t_{0}\right)=0$. Obviously we have $f^{a}\left(t_{1}\right)=1$. Consider the sequence $a^{\prime}$ defined as follows : in the sequence $a$ we delete each 1 read at each occurence of $t_{1}$ and we replace each 0 read at each occurence of $t_{0}$ with a prefix code of the number of occurences of $t_{1}$ before the next occurence of $t_{0}$. The following program computes $a$ using $a^{\prime}$.




The same arguments as in the proof of Lemma 3 enable us to conclude that $a$ is nonrandom.

Conclusion: Since $f^{a}(q)>0, f^{a}\left(t_{0}\right)>0$ and $f^{a}\left(t_{1}\right)>0$ both states $q_{0}$ and $q_{2}$ are frequent.

Step by step we show that any state reachable from $q$ is frequent and thus, according to Lemma 3 , is complete. Such a state is recurrent. Therefore, the class of $q$ is absorbing and complete.

The following lemma is an immediate corollary of Lemma 4 and of the fact that a complete absorbing class accepts any sequence.

Lemma 5 For all random sequence $a, T$ accepts $a$ if and only if $T$ reaches on a one of its complete absorbing classes.

\section{Decidability of the equality of letter to letter rational transducers on random sequences}

We say that two transducers $T_{1}$ and $T_{2}$ are equal on a sequence $s$ if $T_{1}(s)=T_{2}(s)$. Note the difference between this definition and the definition of the equivalence of transducers : $T_{1}$ and $T_{2}$ are equivalent if $T_{1}(w)=T_{2}(w)$ for all word $w$. The equality of a class $\Theta$ of rational transducers on a class $S$ of sequences is decidable if there is an algorithm that decides, for any transducers $T_{1}$ and $T_{2}$ in $\Theta$, and any sequence $s$ in $S$ spelled letter by letter, whether $T_{1}(s)=T_{2}(s)$, using a finite prefix (of unknown length) of $s$.

$$
\exists A \forall\left(T_{1}, T_{2}\right) \in \Theta^{2} \forall s \in S \begin{cases}A\left(T_{1}, T_{2}, s\right)=y e s & \text { if } T_{1}(s)=T_{2}(s) \\ A\left(T_{1}, T_{2}, s\right)=n o & \text { otherwise }\end{cases}
$$

If $\Theta$ is the set of letter to letter rational transducers and $S$ is the set of all sequences, the equality is not decidable. Consider transducers $T_{1}$ and $T_{2}$ defined in Figure 2. Suppose

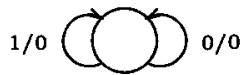

$T_{1}$

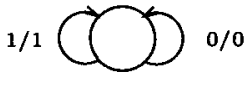

$\boldsymbol{T}_{\mathbf{2}}$

Figure 2: Two transducers equal on $0^{\infty}$ and not equal on $0^{n} 10^{\infty}$

there is an algorithm $A$ deciding, for any sequence $s$, whether both transducers $T_{1}$ and $T_{2}$ are equal on $s$. Let $s_{1}=0^{\infty}$. Since $T_{1}\left(s_{1}\right)=T_{2}\left(s_{1}\right), A\left(T_{1}, T_{2}, s_{1}\right)$ halts having read a prefix of length $n$ of $s_{1}$ and outputs yes. Now, let $s_{2}=0^{n} 10^{\infty}$. Since the $n$ 
first letters of $s_{1}$ and $s_{2}$ are the same, $A\left(T_{1}, T_{2}, s_{2}\right)$ halts and outputs yes, although we obviously have $T_{1}\left(s_{2}\right) \neq T_{2}\left(s_{2}\right)$.

However, the equality becomes decidable if we restrict ourselves to random sequences. In order to show this result, we need to define the notion of product transducer.

Definition 5 Let $T_{1}$ and $T_{2}$ be two transducers. Their product transducer $T=T_{1} \times T_{2}$ is defined as follows : if we can reach $q_{1}^{\prime}$ from $q_{1}$ in the transducer $T_{1}$ and $q_{2}^{\prime}$ from $q_{2}$ in the transducer $T_{2}$, with the same letter $b_{\text {in }}$ in input and the same letter $b_{o u t}$ in output, then $T$ contains a transition between $\left(q_{1}, q_{2}\right)$ and $\left(q_{1}^{\prime}, q_{2}^{\prime}\right)$ reading $b_{i n}$ and outputting bout.

The product transducer $T=T_{1} \times T_{2}$ accepts a sequence $a$ if and only if $T_{1}$ and $T_{2}$ accept $a$ and output the same sequence.

Theorem 1 The equality of letter to letter rational transducers on random sequences is decidable.

Proof Let $a$ be a random sequence. $T_{1}$ and $T_{2}$ accept $a$ and output the same sequence if and only if their product transducer $T$ accepts $a$ or, according to Lemma 5 , if and only if $T$ reaches on $a$ one of its complete absorbing classes. If $T$ halts, we distinguish there are different cases :

- $T_{1}$ and $T_{2}$ accept $a$ and output different sequences and thus $T_{1}$ and $T_{2}$ are not equal on $a$;

- one transducer accepts $a$ and the other rejects $a: T_{1}$ and $T_{2}$ are not equal on $a$;

- $T_{1}$ and $T_{2}$ rejects $a:$ if the output words are the same $T_{1}$ and $T_{2}$ are equal on $a$, otherwise they are not.

Finally, $T_{1}$ and $T_{2}$ are equal on $a$ if and only if either $T$ reaches on $a$ one of its complete absorbing classes or $T, T_{1}, T_{2}$ halt simultaneously with the same output words. These observations lead to the following decision algorithm :

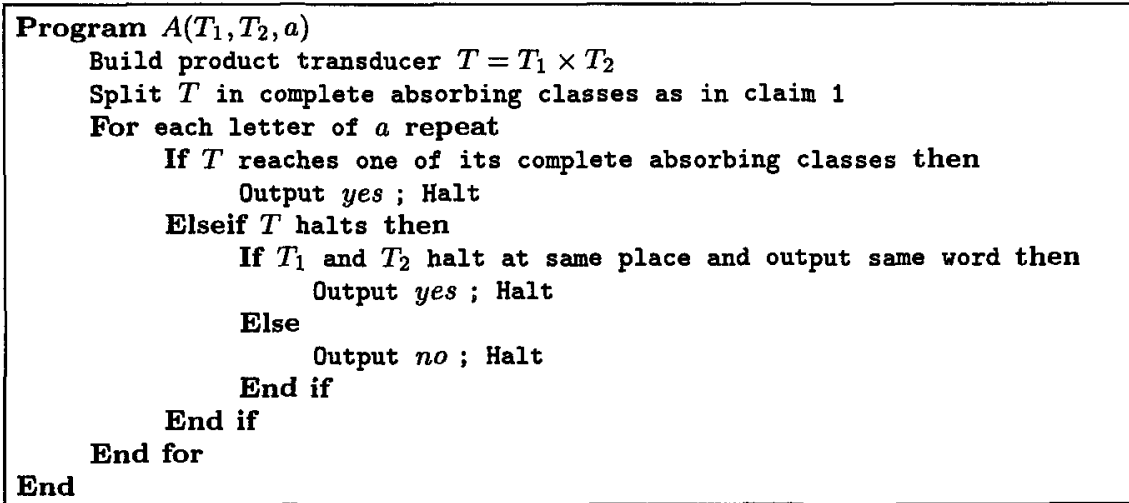




\section{Classification of images of random sequences}

In this part we are interested in the images of random sequences by letter to letter rational transducers. Theorem 2 shows the influence of the transducer's structure on the sequence we obtain.

Definition 6 A state $q$ is partially indifferent if there are two different input words having the same length, leading to a same state from $q$ and having the same image.

Definition $7 A$ state $q$ is discriminating if there exists $l>0$ satisfying the following property : from $q$, the images of two words $w_{1}$ and $w_{2}$ of length $l$ end with same letter if and only if $w_{1}$ and $w_{2}$ start with same letter.

Theorem 2 Let $T$ be a letter to letter rational transducer, let a be a random sequence accepted by $T$ and let $s$ denote $T(a)$.

1. if $T_{a}$ does not contain any partially indifferent state then $s$ is random;

2. (a) if $T_{a}$ contains at least one partially indifferent state then $s$ is non-random. Moreover:

(b) if $T_{a}$ contains a discriminating state then $s$ is non-recursive ;

(c) if $T_{a}$ does not contain any discriminating state, then $s$ is recursive and periodic : $s=u v^{*}$.

Remark 1 Let $Q$ denote the number of states of a transducer $T$. We can show that the existence of a partially indifferent state in $T$ is decidable in a time $\mathcal{O}\left(Q^{2}\right)$ and the existence of a discriminating state in $T$ is decidable in a time $\mathcal{O}\left(2^{2 Q}\right)$.

Example 1 Call a transducer one-to-one if it defines an injective mapping on (infinite) sequences. A one-to-one transducer does not contain any partially indifferent state. The transducer of Figure 3 is a non-trivial example of a one-to-one transducer, that transforms a sequence $a$ in $0 a$. It is non-trivial because it is not just a injective morphism of words.

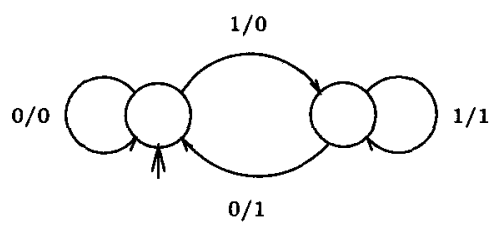

Figure 3: A non trivial one-to-one transducer 
Example 2 Injectivity is not a necessary condition to map random sequences on random sequences. Consider the transducer $T$ of Figure 4 . Since sequences $0^{\infty}$ and $10^{\infty}$ have the same image $01^{\infty}, T$ is not a one-to-one transducer. However, $T$ does not contain any partially indifferent state since there is no state reachable from two transitions having same output letter.

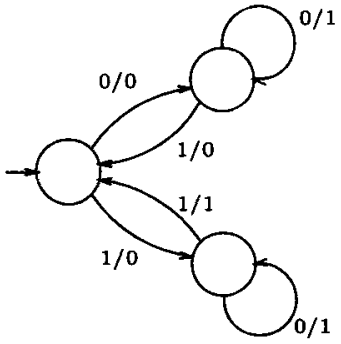

Figure 4: A many-to-one transducer without partially indifferent states

\subsection{Proof}

Proof of 1. and 2.(a) We have to show that $s$ is random if and only if $T_{a}$ does not contain any partially indifferent state.

Only if : We prove that if $T_{a}$ contains at least one partially indifferent state $q$ then $s$ is non-random.

Since $q$ is a partially indifferent state, there are two words $w_{1}$ and $w_{2}$ leading to the same state $q^{\prime}$ from $q$ and outputting the same word $u$. Let $l$ be the length of $w_{1}$ and $w_{2}$. Let $f$ be a coding function from $\{0,1\}^{l}$ to $\{0,1\}^{l}$ such that $f\left(w_{1}\right)$ and $f\left(w_{2}\right)$ differ only in their last letter. For example let $f\left(w_{1}\right)=v 0$ and $f\left(w_{2}\right)=v 1$, where $v$ is a word of length $l-1$. Let $a^{\prime}$ be defined as follows : in the sequence $a$ we replace each word $w$ of length $l$ read from state $q$ with $v$ if $w=w_{1}$ or $w=w_{2}$, with $f(w)$ otherwise. The following program computes $s$ with $a^{\prime}$ as an input.

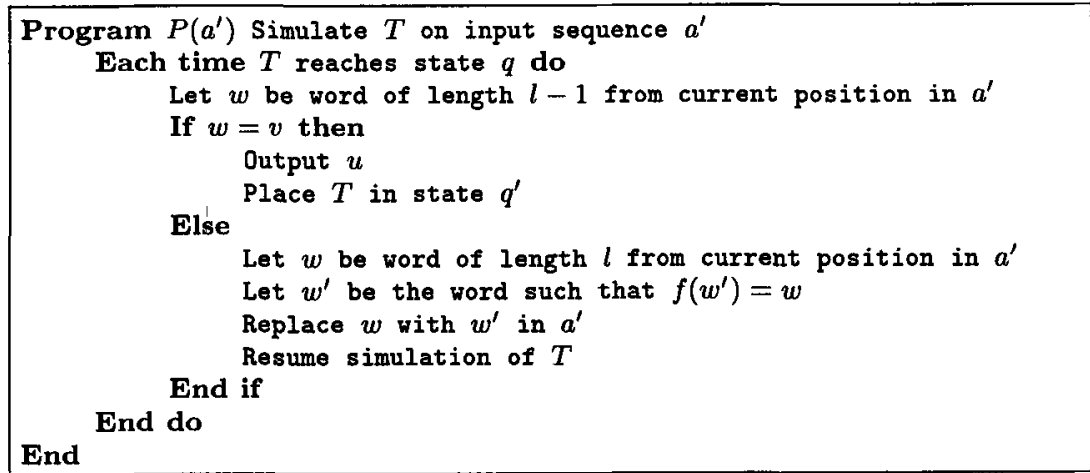


The same arguments as in the proof of Lemma 3 enable us to conclude that $s$ is nonrandom.

If : We prove that if $T_{a}$ does not have any partially indifferent state then $s$ is random. Suppose that $s$ is non-random. We show that this implies that $a$ is non-random too.

Let $u(i, j)$ denote a recursive function mapping pairs of integers to words. Let $\Gamma_{u(i, j)}$ denote the set of all sequences having the prefix $u(i, j)$, let $\Omega_{i}$ denote $\bigcup_{j} \Gamma_{u(i, j)}$ and let $\mu$ denotes uniform measure on the set of all sequences. Since $s$ is non-random, $s$ belongs to a constructive null set, i.e. there is a recursive function $u(i, j)$ such that :

1. $\forall i \mu\left(\Omega_{i}\right) \leq 2^{-i}$

2. $\forall i s \in \Omega_{i}$

Note that we can suppose that, given $i$, all sets $\Gamma_{u(i, j)}, j \in \mathbb{N}$ are disjoint. We will define a function $\hat{u}(i, j)$ mapping pairs of integers to words such that for all $i$ we have $\{T(\hat{u}(i, j)) \mid j \in \mathbb{N}\}=\{u(i, j) \mid j \in \mathbb{N}\}$. The following program $P(i, j)$ computes $\hat{u}(i, j)$ using the recursive function $u(i, j)$ and the transducer $T$.

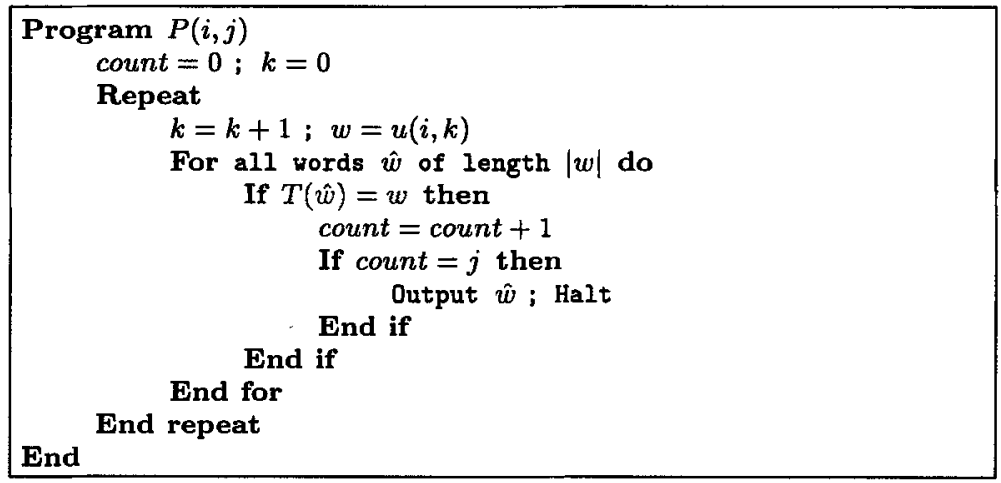

Since $u(i, j)$ is recursive, $\hat{u}(i, j)$ is recursive too. Let $\hat{\Omega}_{i}=\bigcup_{j} \Gamma_{\hat{u}(i, j)}$. Since $\hat{u}(i, j)$ is the preimage of a $u(i, k), a$ belongs to $\hat{\Omega}_{i}$ for all $i$. Moreover we have :

$$
\forall i \mu\left(\hat{\Omega}_{i}\right) \leq \sum_{j} \mu\left(\Gamma_{\hat{u}(i, j)}\right)
$$

Let $Q$ be the number of states of $T$. Since $T_{a}$ does not contain any partially indifferent state there are at most $Q$ different words having the same image. Thus we have :

$$
\begin{aligned}
\forall i \mu\left(\hat{\Omega}_{i}\right) & \leq \sum_{j} Q \mu\left(\Gamma_{u(i, j)}\right) \\
& =Q \mu\left(\Omega_{i}\right) \\
& \leq Q 2^{-i}
\end{aligned}
$$

Hence $a$ belongs to a constructive null set defined by $\hat{u}(i, j): a$ is non-random. 
Proof of 2.(b) Let $q$ be a discriminating state. There exists $l>0$ such that, observing the last letter of the image of an input word of length $l$ from $q$, we can retrieve the first letter of this input word. Assume for example that all words of length $l$ starting with 0 (respectively with 1 ) have their images ending with 0 (respectively with 1 ).

Suppose $s$ is recursive. Let $P$ be a program that computes $s$. Consider the sequence $a^{\prime}$ defined as follows : in the sequence $a$ we delete letters read at each occurence of $q$. The following program computes $a$ using $P, T$ and $a^{\prime}$. Let $q_{0}$ (respectively $q_{1}$ ) denote the state reached when 0 (respectively 1 ) is read at state $q$.

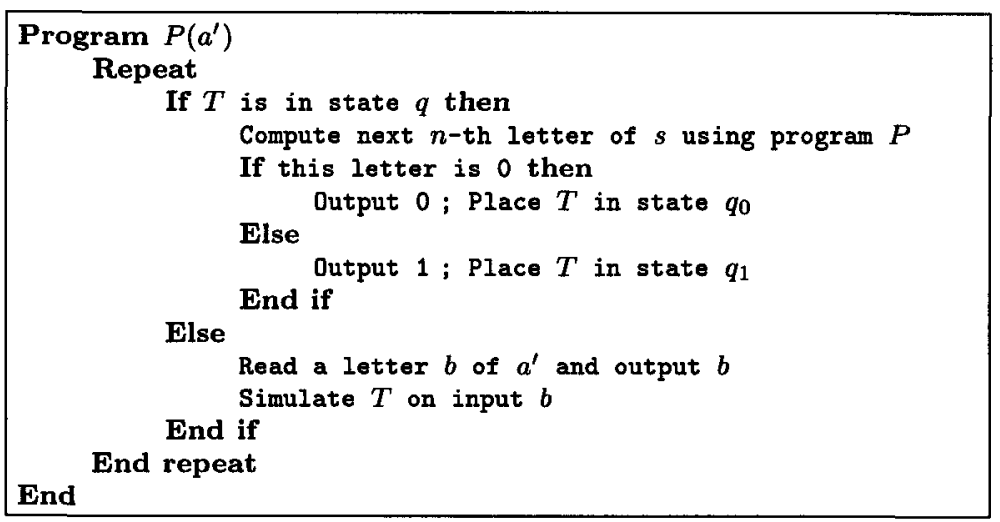

The same arguments as in the proof of Lemma 3 enable us to conclude that $a$ is nonrandom.

Proof of 2.(c) Firstly we prove the following property : for all state $q$ of $T_{a}$, for all $l$, for all words $w_{1}$ and $w_{2}$ of length $l$, the images of $w_{1}$ and $w_{2}$ from $q$ are the same. We use a recurrent reasoning on $l$ to show this. $W(q, l)$ denotes the set of image words of length $l$ from $q$.

Step 1: The property is true for $l=1$. Indeed each state outputs the same letter whatever the input letter is, since each state of $T_{a}$ is not discriminating.

Step 2: Let $q$ be a state of $T_{a}$. Let $q_{0}$ (respectively $q_{1}$ ) be the state reached from $q$ when 0 (respectively 1 ) is read. Suppose the property is true for all $l \leq L$. Thus all words of $W\left(q_{0}, L\right)$ (respectively $W\left(q_{1}, L\right)$ ) end with the same letter $b_{0}$ (respectively $b_{1}$ ). Since $q$ is not discriminating, we necessarily have $b_{0}=b_{1}$. Thus all input words of length $L+1$ have images ending with same letter from $q$.

We prove now that $s$ is periodic, i.e. there are two words $u$ and $v$ such that $s=u v^{*}$. Let $a^{\prime}$ be the suffix sequence of $a$ read from the first occurence of a recurrent state $q$. According to the previous property, we have $q\left(a^{\prime}\right)=q\left(0^{*}\right)$. Since the automaton is finite and deterministic, there is necessary a loop. 


\subsection{From an arithmetical point of view}

The following theorem is an application of Theorem 2 within the frame of arithmetic. We call 'random real' a real of which the binary expansion is a random sequence. We remind of the reader that a real is algebraic if it is the root of a polynomial equation having integer coefficients. A real is transcendental if it is not algebraic.

Theorem 3 The image of a random real by a letter to letter rational transducer is either rational or transcendental.

Proof Let $a$ be the binary expansion of the image of a random real. If $a$ has a finite complexity then it is periodic and then the real number is rational. If $a$ has an infinite complexity then the real number is transcendental. Indeed, since an algebraic number is a root of a polynomial equation with integer coefficients, it has a finite complexity : we can give more and more accurate approximations of this root using an algorithm.

\section{Open problems}

In this first approach we have only considered letter to letter rational transducers. We should study whether the results we have obtained remain true in the general case. Moreover we think that links between simple and 'pure' algebraic objects (rational transducers) and 'pure' complex objects (random sequences), and also the links with Markov chains, should be study thoroughly.

\section{References}

[Cal94] C. Calude. Information and Randomness, an Algorithmic Perspective. Springer-Verlag, 1994.

[CL88] M. Chrobak and M. Li. $k+1$ heads are better than $k$ for PDAs. J. Comput. Syst. Sci., $37: 144-155,1988$.

[Lev73] L.A. Levin. On the notion of random sequence. Soviet Math. Dokl., 14 :1413$1416,1973$.

[LV93] M. Li and P. Vitányi. A new approach to formal language theory by Kolmogorov's complexity. SIAM J. Comput., 24 :398-410, 1993.

[LV97] M. Li and P. Vitányi. An Introduction to Kolmogorov Complexity and its Applications. Springer-Verlag, 1997.

[ML66] P. Martin-Löf. The definition of random sequences. Inform. Contrib., 9 :602619, 1966. 
[MS97] A. Mateescu and A. Salomaa. Aspect of classical language theory. In Handbook of Formal Languages, volume 1, pages 175-251. Springer-Verlag, 1997.

[MT96] S.P. Meyn and R.L. Tweedie. Markov Chains and Stochastic Stability. Springer-Verlag, 1996.

[Niv68] M. Nivat. Transductions de langages de chomsky. Annales de l'Institut Fourier, $18: 339-455,1968$.

[PD97] S. Porrot and M. Dauchet. Discrete curves complexity. Rapport interne, LAIL, 1997.

[Sch73] C.P. Schnorr. Process complexity and effective random tests. J. Comput. System Sci., 7 :376-388, 1973.

[Sei86] J. Seiferas. A simplified lower bound for context-free-language recognition. Inform. Contrib., 69 :255-260, 1986.

[Tho90] W. Thomas. Automata on infinite objects. In Handbook of Theoretical Computer Science, volume B, pages 133-191. Elsevier, 1990. 